\section{Pathogenic Variation of Cladosporium caryigenum Isolates and Corresponding Differential Resistance in Pecan}

\section{Patrick J. Conner}

Department of Horticulture, University of Georgia, Coastal Plain Experiment Station, 4604 Research Way, Tifton, GA 31793

Katherine L. Stevenson

Department of Plant Pathology, University of Georgia, Athens, GA 30602-7274

Additional index words. Carya illinoinensis, scab, vertical resistance, fungus, histology

Abstract. To facilitate the breeding of scab-resistant pecan [Carya illinoinensis (Wangenh.) C. Koch] cultivars, more information is needed about the pathogenic variation of the causal organism, Cladosporium caryigenum (Ell. et Lang.) Gottwald (1982). This study examined the virulence patterns resulting from the field inoculations of 19 pecan cultivars with 12 monoconidial isolates from 8 pecan cultivars at 7 locations. The virulence pattern was different for each of the fungal isolates. Each isolate was virulent on some cultivars and avirulent on others. Most isolates were most virulent on the cultivar of their origin and one or a few other cultivars. Several cultivars were resistant to most of the tested isolates. The results indicate a large pool of differential and ephemeral resistance to scab resides in the pecan germplasm. Microscopic examination of developing lesions suggests that resistance is mediated by the speed of plant cell wall modifications that limit the growth of subcuticular hyphae. This information will be useful in the selection of cultivars with functionally different resistance genes for use in designing cultivar mixtures or in breeding programs.

The pecan scab fungus is the most economically damaging pathogen of pecan in the humid growing conditions of the southeastern U.S. Foliar infections result in black circular lesions that under favorable conditions can result in severe leaf spotting, premature defoliation, and shoot death. Development of lesions on fruit shucks reduces yield and nut quality, and if not controlled it can result in total crop loss (Sanderlin, 1994). Commercial pecan plantings may require up to 11 fungicide applications annually to control the disease (Ellis et al., 2000). Pecan scab has developed resistance to at least one common fungicide, benomyl (Sanderlin, 1992), and recent concerns over negative environmental health effects of pesticides has increased pressure to restrict valuable control agents (Guillebeau, 1998). The development of scab resistant cultivars with excellent commercial quality would greatly increase the profitability of pecan cultivation in the southeastern U.S. and it is the focus of several cultivar development programs (Conner, 1999; Goff et al., 1998; Thompson and Grauke, 1994).

Effective breeding for resistance to Cladosporium caryigenum requires information on the pathogenic diversity of the fungus. Many cultivars that were once highly resistant to scab are now widely considered susceptible. For example, 'Desirable' and 'Stuart' are grown throughout the Southeast and were initially popular in part due to their high levels of scab

Received for publication 24 Feb. 2003. Accepted for publication 23 June 2003.

${ }^{1}$ Corresponding author; e-mail pconner@tifton. cpes.peachnet.edu. resistance. Unfortunately, this resistance has collapsed and now both cultivars are considered susceptible. The appearance and spread of a race of the fungus capable of infecting 'Stuart' has been documented in the literature (Cole and Gossard, 1956).

The impermanence of cultivar resistance to scab infections can be explained by variation in populations of $C$. caryigenum. Differences in virulence among scab isolates were first reported by Demaree and Cole (1929) who used orchard inoculations to demonstrate that at least four races of the pathogen existed that differed in their ability to infect pecan cultivars. Further testing demonstrated the presence of at least four races based on their pathogenicity on four pecan cultivars in the greenhouse and the field (Converse, 1960). Bracewell (1996) found cultivar-inoculum source interaction was significantly associated with disease intensity. Using a detached leaf assay, Conner (2002) examined microscopically four $C$. caryigenum isolates from four cultivars to determine the susceptibility of each cultivar to the four isolates. Isolates differed in their ability to form subcuticular hyphae on the different cultivars, with the greatest amount of infection usually occurring when the isolate was placed back onto the cultivar from which it was isolated.

It is apparent from these studies that a range of genetic or pathotypes of $C$. caryige num exist that differ markedly in their ability to cause disease on different pecan cultivars. The work reported here was undertaken to further examine the extent of pathogenic variation among scab isolates using a larger number of cultivars and fungal isolates. These results may be useful in designing crosses to pyramid resistance genes into a single cultivar or in selecting combinations of cultivars to be included in an orchard.

\section{Materials and Methods}

Inoculum preparation. Twelve isolates from eight pecan cultivars and seven locations were used in the study (Table 1). Frozen stocks of monoconidial scab isolates were prepared according to Conner (2002). Isolates used in the experiment were revived from frozen stocks by placing filter paper discs with the dried germinated conidia directly onto oatmeal agar plates. After 2 weeks of incubation at $24^{\circ} \mathrm{C}$ in the dark, the fungal colonies were homogenized in sterile water and spread across a fresh oatmeal agar plate that was again incubated for 2 weeks at $24^{\circ} \mathrm{C}$. Subsections of this plate were homogenized in water and spread onto oatmeal agar plates to produce conidia for inoculations After 1 to 2 weeks growth at $24{ }^{\circ} \mathrm{C}$, conidia were harvested by flooding cultures with $2 \mathrm{ml}$ of sterile water and the concentration adjusted to $1 \times 10^{6}$ conidia $/ \mathrm{mL}$.

Inoculation. The cultivars chosen for this study represent a wide range of currently popular or historically important cultivars. Trees were grown in an orchard located at the Coastal Plain Experiment Station in Tifton, Ga All trees received recommended fertilization and supplemental irrigation (Crocker, 1996). No fungicides were applied to the orchard during the course of this experiment.

Inoculations were made by spraying expanding shoots with a conidial suspension of C. caryigenum until runoff using a spray bottle. Each shoot consisted of five to ten compound leaves and each leaf consisted of 5 to 13 leaflets

\begin{tabular}{lccc}
\multicolumn{5}{l}{ Table 1. Sources of isolates of Cladosporium caryigenum used in study. } \\
\hline Isolate & Year & Host cultivar & Location $^{\mathrm{a}}$ \\
\hline Cf-Au-2 & 1999 & Cape Fear & Lee, Ala. \\
De-Tif-3 & 1999 & Desirable & Tift, Ga. \\
De-Mo-11 & 1999 & Desirable & Jefferson, Fla. \\
AL-LI-DE & 2000 & Desirable & Lee, Ala. \\
GA-FW-DE & 2000 & Desirable & Dougherty, Ga. \\
LA-MP-DE & 2000 & Desirable & Natchitoches Par, La. \\
Fa-Tif-11 & 2001 & Farley & Tift, Ga. \\
Ma-Tif-11 & 2001 & Mahan & Tift, Ga. \\
Pa-OK-11 & 2000 & Pawnee & Payne, Okla. \\
Sc-Tif-1 & 1999 & Schley & Tift, Ga. \\
St-Tif-13 & 2001 & Stuart & Tift, Ga. \\
Wi-Tif-2 & 1999 & Wichita & Tift, Ga. \\
\hline
\end{tabular}




\section{Plant Pathology}

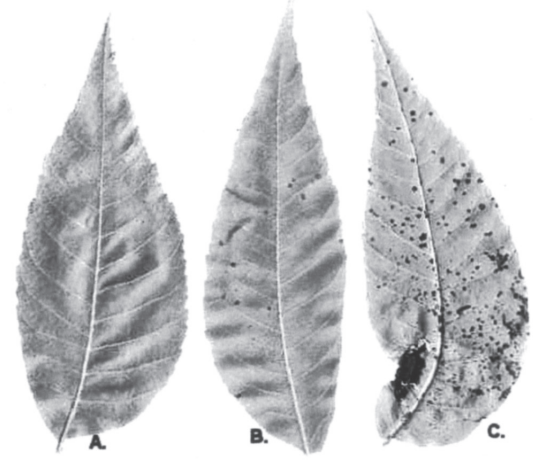

Fig. 1. Typical symptoms induced by artificial inoculation of Cladosporium caryigenum on differen pecan hosts. (A) Isolate De-Tif-3 on the cultivar 'Mahan' showing a type 2 intermediate reaction with pin-point lesions with sparse or no sporulation. (B) Isolate De-Tif-3 on 'Cape Fear' showing a type 3 intermediate reaction with chlorotic lesions with limited expansion and sporulation.

(C) Isolate De-Tif-3 on 'Desirable' showing a type 4 susceptible reaction with large expanding lesions and abundant sporulation.

that varied in maturity from most mature at the base to newly formed leaflets at the tip. Thus, for each inoculated shoot a minimum of 20 leaflets were still expanding and susceptible to infection at the time of inoculation. Leaves were then wrapped loosely with a moist paper towel and enclosed in aluminum foil to reflect sunlight. Leaves were covered for $48 \mathrm{~h}$ and then uncovered to dry. The tested cultivars always included the original cultivar host for each isolate. This served as a general control to assure that the inoculum was viable and virulent. Noninoculated control shoots

\section{Results and discussion}

were treated in the same manner except that leaves were sprayed with sterile water. No lesions were produced in any of the control inoculations so this data was omitted from the results. Leaves were collected $28 \mathrm{~d}$ after inoculation and all leaflets were examined for the presence of scab lesions. Disease severity was assessed on individual leaflets on a scale of 1 to 4 , where 1 = no visible symptoms, 2 $=$ pin-point necrotic lesions with sparse or no sporulation, $3=$ chlorotic lesions with limited expansion and sporulation, and $4=$ large dark expanding lesions with abundant sporulation. Shoots were scored as the most severe rating observed on any leaflet. The entire experiment was repeated for a total of two trials for each cultivar-isolate combination.

Microscopic examination. For microscopic examination of disease progress, four isolates of $C$. caryigenum, Fa-Tif-11, De-Tif-3, PaOK-11, and Wi-Tif-2, were used to inoculate leaves of 'Farley' in the field using the methods described above. Whole leaf mounts were used to observe disease progression over a 2-week period. A $1-\mathrm{cm}^{2}$ section was cut from the center of the leaflet for each sample. Samples were stained with trypan blue and chlorazol Black E according to Conner (2002). Leaves were sampled at $2,4,7,11$, and 15 d postinoculation. Eight leaflets for each isolate / time period were examined. On each sample 25 germinated conidia were examined for the presence of subcuticular hyphae, reproductive initials, and halo staining of the nearby epidermal cells. Thus, 200 germinated conidia were observed for each data point. Differences in fungal growth among $C$. caryigenum isolates at each time period were determined statistically by one way analysis of variance (SigmaStat).
Field inoculation of pecan trees is chal lenging because of the large plant size and the requirement for free moisture during the initial infection period (Turechek and Stevenson, 1998). Greenhouse inoculation were not attempted due to the difficulty of obtaining plant material of suitable size and because greenhouse inoculations often do not sporulate as readily as field inoculations (Converse, 1960).

Inoculations produced a range of symptoms on host leaves varying from small pinpoint lesions to the large spreading dark lesions typical of natural scab infections (Fig. 1). Disease reactions were categorized based on the most severe reaction observed on any leaflet on the treated shoot. This avoided the bias that could be introduced by the beginning of ontogenic resistance in the more mature basal leaflets or by inoculum falling off some leaves resulting in a shifting of the score to the lower end of the scale. By consistently taking the worst case observed, the results were biased towards the hypothesis of all cultivars susceptible to all isolates. However, reaction scores were relatively consistent between replicate inoculations with the majority of ratings being equal and differences usually separated by only a single class (Table 2).

Leaves producing no visible symptoms were considered to be resistant to the challenging isolate. Leaves producing a type 4 reaction, with its typical expanding lesion, abundant sporulation, and surrounding green host tissue were considered susceptible to the challenging isolate. Reaction types falling between these two extremes were categorized

Table 2. Symptoms ${ }^{\mathrm{z}}$ caused by differential isolates of Cladosporium caryigenum on pecan cultivars.

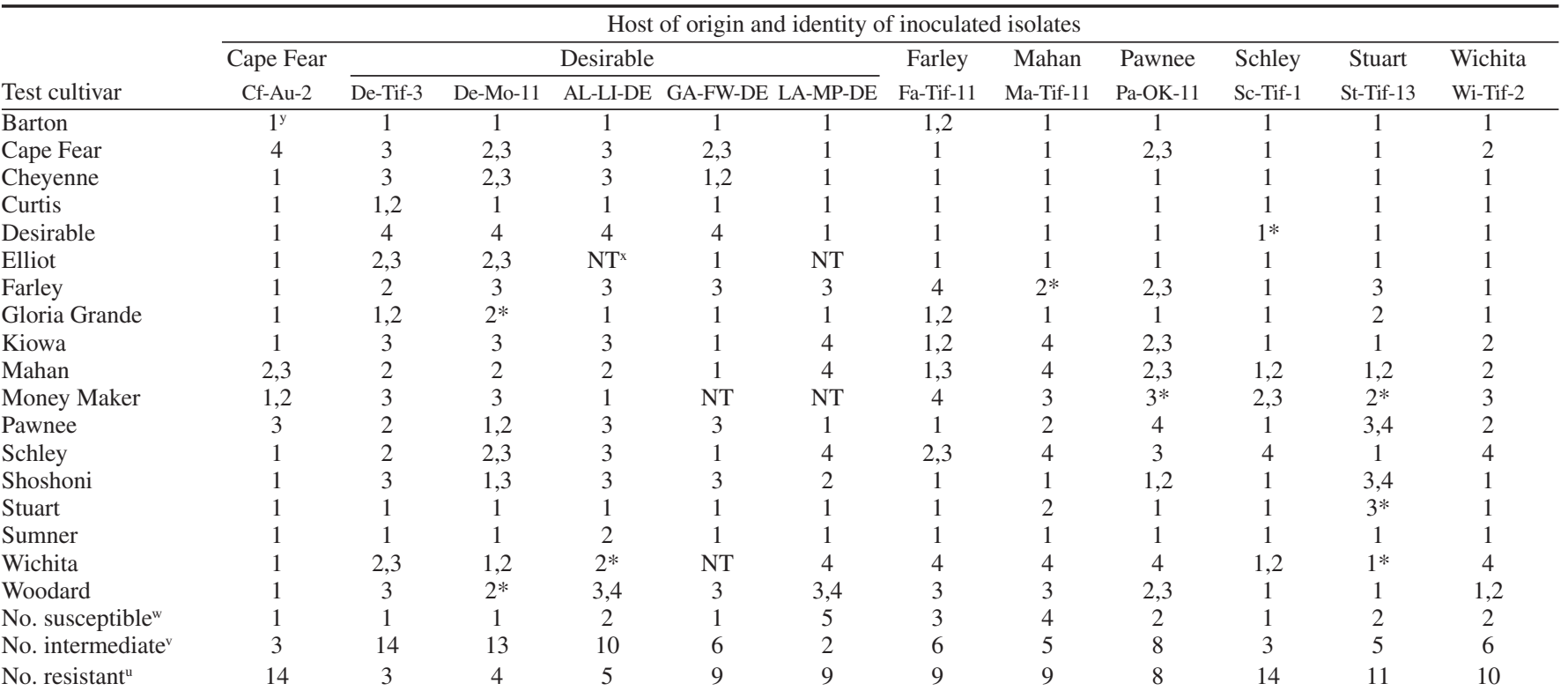

"Symptoms: 1 = no visible symptoms; 2 = pinpoint lesions with no or sparse sporulation; $3=$ chlorotic lesions with reduced expansion and sporulation; $4=$ large spreading lesions with abundant sporulation. Shoots were scored as the most severe expression of symptoms found on any of the leaves.

yTwo replications were performed for each entry, except where the entry is followed by an *, indicating a single replication. Identical results are indicated by a single number, discrepancies are indicated by listing both results.

${ }^{x}$ Not tested.

"Number of cultivars inoculated with each isolate with no visible disease symptoms (rating of 1 ).

"Number of cultivars inoculated with each isolate showing a 2 or 3 reaction.

"Number of cultivars inoculated with each isolate showing a 4 reaction in at least one test. 


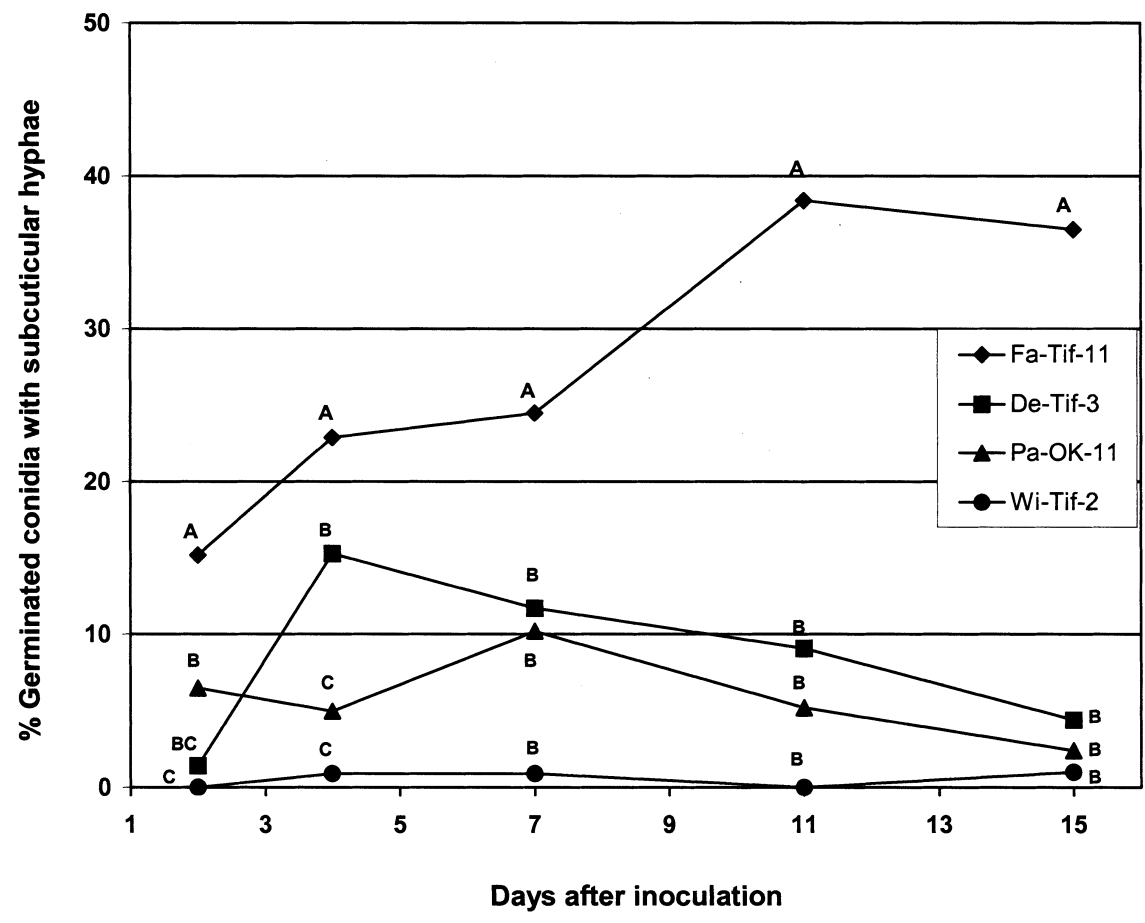

Fig. 2. Microscopic examination of 'Farley' pecan leaves inoculated with four Cladosporium caryigenum isolates showing variation in the percent germinated conidia producing subcuticular hyphae. Each data point represents the average of eight replicate leaves. Data points for each time period with a different letter were significantly different, $P<0.05$

as intermediate. Type 2 lesions produced pinpoint necrotic flecks and only rarely sporulated.

Type 3 lesions had restricted lesion growth, reduced sporulation, and were surrounded by a ring of chlorotic host tissue. A similar range of symptoms has been reported in the literature. Converse (1960) categorized lesions into normal, yellow-margined, and fleck types. Bracewell (1996) reported that when 'Wichita' and 'Sumner' isolates were cross-inoculated onto the other cultivar, lesions were smaller, surrounded by a chlorotic halo, and had restricted sporulation. Turechek and Stevenson (1998) found that inoculum from 'Wichita' trees produced smaller, yellow to light brown lesions with significantly fewer spores per lesion and per lesion unit area on 'Sumner' leaves than they did when inoculated onto 'Wichita' leaves. It thus appears to be common for cross-inoculations to produce atypical lesions with reduced size and conidia production. Because resistance ranking order is dependent upon the scab isolate tested (Table 2 ) and a range of reaction types was observed, resistance can be classified as vertical partial resistance (Vanderplank, 1984).

All cultivars showed a differential susceptibility to the isolates (Table 2). 'Barton', 'Curtis', and 'Sumner' were resistant to most isolates, producing only a single type 2 reaction to one of the isolates. These three cultivars are generally considered to have good field resistance to scab (Sparks, 1992). Conversely, 'Wichita' and 'Schley', cultivars rarely used for new plantings because of their scab susceptibility (Sparks, 1992), were susceptible to several of the isolates. However, 'Cheyenne', a cultivar that is very susceptible in the field, showed good resistance to most of the isolates. Thus the isolates tested in this study are not sufficient to accurately determine the field

Isolates appear to have a narrow range of cultivars on which they are most virulent. Five isolates only produced a susceptible (type 4) reaction on one cultivar, four produced a susceptible reaction on two cultivars, and the remaining three isolates produced a susceptible reaction on three, four, or five cultivars. Generally, isolates were most virulent on their cultivar of origin. The only exceptions to this were isolate St-Tif-13, obtained from a 'Stuart' tree, which produced an intermediate reaction when inoculated back onto 'Stuart', and isolate LA-MP-DE, obtained from a 'Desirable' tree, which did not produce any visible reaction when inoculated onto 'Desirable'. St-Tif-13 may represent an isolate obtained from a cultivar to which it is only partially adapted, as it was able to sporulate on 'Stuart' That LA-MP-DE did not produce any visible symptoms on 'Desirable' is more difficult to explain. However, several other cultivars were susceptible to this isolate suggesting that the tree from which it was obtained may have been misidentified in the orchard. Often when isolates produced a type 4 reaction on several cultivars those cultivars were related. For example, Ma-Tif-11 and LA-MP-DE both produced type 4 reactions on 'Kiowa', 'Mahan', 'Schley', and 'Wichita'. All four cultivars are closely related as 'Mahan' is likely a self of 'Schley', and is a parent of 'Kiowa', and 'Wichita'.

The number of cultivars tested in this study resistant to each isolate varied from 3 susceptibility of all cultivars. to 14. In general, most isolates were not able to produce visible symptoms on about half of the tested cultivars. Notable exceptions to this were the three 'Desirable' isolates De-Tif-3, De-Mo-11, and Al-LI-DE that produced intermediate reaction types on 14,13 , and 10 cultivars respectively. However, these isolates only produced susceptible reactions (class 4) on 'Desirable' or 'Desirable' and 'Woodard' in the case of AL-LI-DE. Interestingly, even though 'Stuart' is one of the founding cultivars of the southeastern pecan industry, and accounts for about $1 / 4$ the current pecan acreage (Florkowski et al., 2000), virulence to 'Stuart' was rare among the isolates tested (Table 2). This suggests there may be a form of stabiliz ing selection acting to reduce the number of virulence genes in the pathogen.

Five different 'Desirable' isolates were used to assess the effect of location on pathogen variation. As was noted above, isolate LAMP-DE was likely to have been isolated from a cultivar other than 'Desirable' and so was not considered. The remaining four isolates shared some similarities. Three out of four were most virulent only on 'Desirable' and the remaining isolate AL-LI-DE was most virulent on 'Desirable' and 'Woodard'. Each also had an intermediate reaction on 'Cape Fear', 'Cheyenne', 'Farley', 'Pawnee', and 'Shoshoni' and was avirulent on 'Barton' and 'Stuart'. This suggests that in screening trials designed to provide maximum pathogen variation, preference should be given to isolates from diverse genotypes over diverse locations.

Comparison of these results with previous studies is difficult due to differences in the environment under which the disease was assessed and in the rating systems used. Bracewell (1996) and Turechek and Stevenson (1998) found that inoculum from a 'Wichita' tree produced an intermediate reaction on 'Sumner'. In this study, no visible symptoms were produced on 'Sumner' inoculated with Wi-Tif-2. This variance may be explained by pathogenic differences between the isolates or by differences in the environment as the previous experiments were conducted in greenhouse. Conner (2002) used the isolates De-Tif-3 and Wi-Tif-2 to inoculate the cultivars 'Wichita', 'Desirable', 'Cape Fear', and 'Elliot' in the field. Each isolate only produced scab lesions on its cultivar of origin; however, only normal type 4 lesions were counted and intermediate reactions were not reported.

To determine the basis for the difference in lesion types, 'Farley' was inoculated with four isolates, Fa-Tif-11, De-Tif-3, Pa-OK-11, and Wi-Tif-2, and examined microscopically over a 2-week period. These four isolates produced a range of disease reactions on 'Farley'; no lesions for Wi-Tif-2, a 2 reaction for De-Tif3 , a 2 and a 3 reaction for $\mathrm{Pa}-\mathrm{OK}-11$, to a 4 reaction for Fa-Tif-11 (Table 2).

Fa-Tif-11 produced significantly more subcuticular hyphae at each time period than did the other three isolates (Fig. 2). Percen germinated conidia with subcuticular hyphae increased with time to a maximum of $38 \%$ on day 11 . In contrast, De-Tif-3 increased to a maximum of $15 \%$ at day 4 and $\mathrm{Pa}-\mathrm{OK}-11$ 


\section{Plant Pathology}

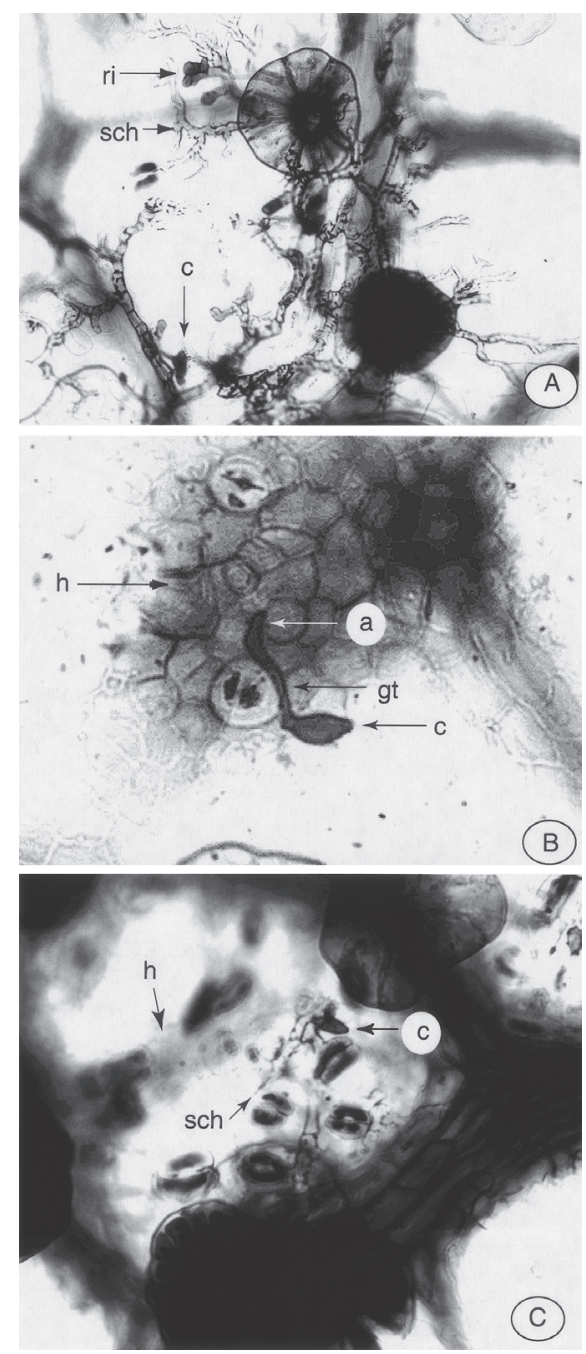

Fig. 3. Development of Cladosporium caryigenum isolates on 'Farley' pecan leaves. (A) Isolate FaTif-11, $11 \mathrm{~d}$ PI showing a extensive subcuticular growth and formation of reproductive initials. (B) Isolate Wi-Tif-2 $4 \mathrm{~d}$ P.I. with darkened halo staining underneath the appressorium. (C) Isolate Pa-OK-11 15 d PI with restricted subcuticular growth surrounded by halo staining. Abbreviations: $\mathrm{a}=$ appressorium, $\mathrm{c}=$ conidium, $\mathrm{gt}=$ germ tube, $\mathrm{h}=$ halo staining, $\mathrm{ri}=$ reproductive initials, sch $=$ subcuticular hyphae.

increased to a maximum of $10 \%$ at day 7 , and both declined steadily to $2 \%$ to $4 \%$ by day 15 . Only a small amount $(\approx 1 \%)$ of subcuticular hyphae were detected at any time period in the Wi-Tif-2 inoculation.

Subcuticular hyphae in each inoculation were monitored for the production of reproductive initials which appear as pairs of melanized cells (Fig. 3A). Reproductive initials in the FATif-11 inoculation were apparent after $11 \mathrm{~d}$ in $8 \%$ of the potential infection sites and nearly doubled to $15 \%$ of the sites by day 15 (Fig. 4). In contrast, reproductive initials were associated with $<1 \%$ of sites in the De-Tif-3 inoculated leaves at day 15 and were not detected at any of the sites in the Pa-OK-11 and Wi-Tif-2 inoculated leaves. These results demonstrate the increased potential for spore production of the susceptible reaction in comparison to the intermediate and resistant reactions.
Conner (2002) found race-specific resistance to be associated with a decreased production of subcuticular hyphae and an increased production of a dark staining halo in epidermal cells directly below the appressorium. Production of a halo around a cell wall penetration or attempted penetration site is a common feature of induced resistance reactions (Moerschbacher and Mendgen, 2000). 'Farley' leaves inoculated with isolate Wi-Tif-2, which

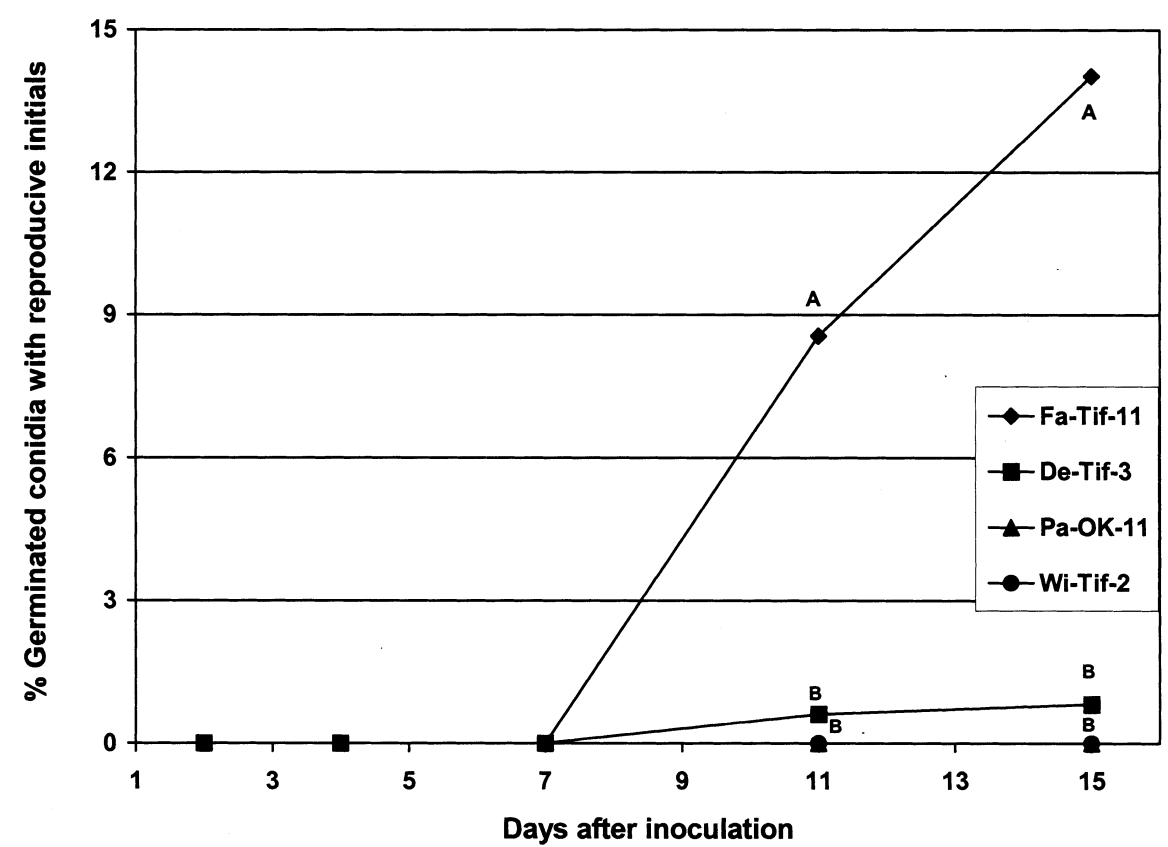

Fig. 4. Microscopic examination of 'Farley' pecan leaves inoculated with four Cladosporium caryigenum isolates showing variation in the percent germinated conidia producing subcuticular growth and reproductive initials. Each data point represents the average of eight replicate leaves. Data points for each time period with a different letter were significantly different, $P<0.05$.

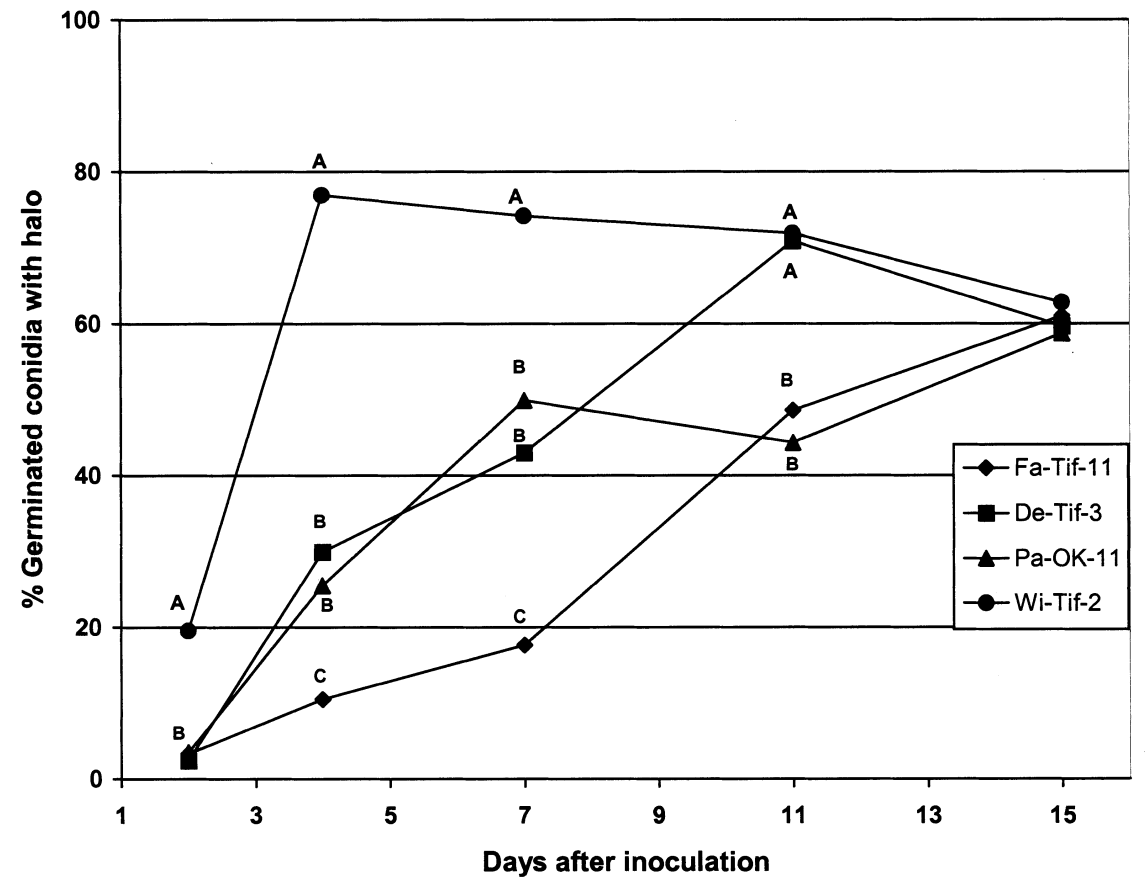

Fig. 5. Microscopic examination of 'Farley' pecan leaves inoculated with four Cladosporium caryigenum isolates showing variation in the percent germinated conidia associated with a halo effect. Each data point represents the average of eight replicate leaves. Data points for each time period with a different letter were significantly different, $P<0.05$. produced no visible symptoms, produced halos at $20 \%$ of the infection sites by day 2 almost $80 \%$ of the infection sites by day which produced intermediate reactions, had halos associated with only $2 \%$ to $3 \%$ of the infections sites at day 2 and $25 \%$ to $30 \%$ of the infection sites at day 4 . Only by day 11 for De-Tif-3 and day 15 for Pa-OK-11 were halos as common as they were in the Wi-Tif- 
2 inoculation. Fa-Tif-11, which produced a susceptible reaction on 'Farley', had the lowest percent of infection sites associated with halo formation. By day 11 it had a similar percentage as Pa-OK-11 and by day 15 all isolates were similar. However, by this time reproductive initials had begun to form (Fig. 4) and hyphal growth was extensive.

Subcuticular hyphae were rarely observed in epidermal tissues showing dark halo staining (Fig. 3C). This staining is likely associated with modifications that slow or prevent fungal breakdown of plant cell walls (Moerschbacher and Mendgen, 2000). The speed of the formation of this modification may be a major factor in the eventual outcome of the reaction. This hypothesis is consistent with the observation that all four isolates had an equal percentage of infection sites associated with halo staining by day 15 , even though quite different fungal growth patterns were observed. A fast halo formation, as was seen in the Wi-Tif-2 inoculation, was associated with an almost complete lack of subcuticular hyphae (Fig. 2) and no visible disease symptoms (Fig. 3B). Isolates De-Tif-3 and $\mathrm{Pa}-\mathrm{OK}-11$, associated with intermediate disease reactions, were slower than Wi-Tif-2 to form halos (Fig. 5). In many cases, the growth of subcuticular hyphae was restricted by dark staining halos (Fig. 3C). In these cases, halo formation may have been sufficiently rapid to reduce lesion size and sporulation, but not enough to prevent evidence of symptoms. In the susceptible reaction with Fa-Tif-11, fungal growth was greatest. Many lesions had begun to form reproductive initials and extensive hyphal growth before any halo formation was seen. Halo formation in these cases was usually restricted to the outer edges of the lesion and seldom encompassed the entire lesion resulting in less restricted hyphal growth.

The reservoir of resistance genes in $C$. il linoinensis seems to be large. Since pecan is dichogamous, new combinations of resistance genes are produced in each generation. This would explain why it is not uncommon for new seedlings to appear to be resistant for several years or until planted on a wide scale, resulting in exposure to a virulent scab strain, which then multiplies rapidly. Ephemeral resistance is difficult to employ effectively in monoculture orchard systems because of the ability of the pathogen to adapt and become virulent on the cultivar over time. This leads to the well known boom and bust cycles encountered with the use of vertical resistance genes in plant breeding (Van der Plank, 1984). The usefulness of a pecan cultivar with a new resistance gene will be largely determined by the durability of resistance and the cultivar's inherent horticultural value. It seems reasonable to assume that most resistance genes will eventually be overcome by the pathogen if the cultivar is widely planted. When the cultivar becomes susceptible, disease must be controlled by fungicide and cultural methods. At this point, the cultivar must have enough horticultural merits to compete with other susceptible cultivars or it will be discarded by the grower.

Durability of resistance may be improved by pyramiding functionally different resistance genes into a single cultivar. This may be an especially effective strategy in dealing with $C$. caryigenum given its apparent lack of a sexual cycle. However, the formation of complex races capable of overcoming several resistance genes may be hastened (Wolfe, 1993). Another strategy would be to incorporate mixtures of cultivars containing different resistance genes into an orchard. Simulation models designed for apple scab predict that a mixture of three different cultivars with different resistance genes could reduce the number of lesions after six generations by $65 \%$ when planted in alternate rows, and by $79 \%$ in a within-row mixture (Gessler and Blaise, 1994). The development of new cultivars with similar horticultural characteristics but different resistance genes would facilitate the adoption of this strategy in pecan orchards.

\section{Literature Cited}

Bracewell, C.J.R. 1996. Pathogenic variation of Cladosporium caryigenum on resistant and susceptible pecan cultivars. MS thesis. Univ. Ga., Athens.

Conner, P.J. 1999. The Georgia pecan breeding program. Proc. S.E. Pecan Growers Assn. 92:77-80.

Conner, P.J. 2002. A detached leaf technique for studying race-specific resistance to Cladosporium caryigenum in pecan. J. Amer. Soc. Hort. Sci. 127:781-785.

Converse, R.H. 1960. Physiologic specialization of Fusicladium effusum and its evaluation in vitro. Phytopathology 50:527-531.

Cole, J.R. and A.C. Gossard. 1956. Stuart pecan found to be susceptible to scab in Mississippi. Plant Dis. Rpt. 40:156.

Crocker, T.F. 1996. Commercial pecan production in Georgia. Univ. Ga. Coop. Ext. Serv. Bul. 609

Demaree, J.B. and J.R. Cole. 1929. Behavior of Cladosporium effusum (Wint.) Demaree on some varieties of pecan. J. Agr. Res. 18:363-370.

Ellis, H.C., P. Bertrand, and T.F. Crocker. 2000 2000 Georgia pecan pest management guide. Univ. Ga. Coop. Ext. Serv. Bul. 841.

Florkowski, W.J., G. Humphries, and T.F. Crocker. 2000. Criteria used by Georgia growers in selecting pecan cultivars-1998 pecan tree inventory. Proc. S.E. Pecan Growers Assn. 93:79-87.

Gessler, C. and P. Blaise. 1994. Differential resistance in apple against scab and its use in breeding and in orchard planting strategies to contro the disease, p. 99-104. In. H. Schmidt and M. Kellerhals (eds.). Progress in temperate fruit breeding. Kluwer, Boston, Mass.

Goff, W.D., M. Nesbitt, R. Mullenax, F. Raspberry, and B. Graves. 1998. Pest-resistant cultivars as a way to reduce input costs. Pecan S. 31(7):6-9.

Gottwald, T.R. 1982. Taxonomy of the pecan scab fungus Cladosporium caryigenum. Mycologia 74:382-390.

Guillebeau, P. 1998. What to do about the food quality protection act? Or how can we protect the pesticides we need? Proc. S.E. Pecan Growers Assn. 91:65-69.

Moerschbacher, B. and K. Mendgen. 2000. Structura aspects of defense, p. 231-277. In. A. Slusarenko, R.S.S. Fraser, and L.C. van Loon (eds.). Mechanisms of resistance to plant diseases. Kluwer, Boston, Mass.

Sanderlin, R.S. 1992. Survey of Louisiana pecan orchards for scab pathogen populations toleran to benomyl fungicide. Proc. S.E. Pecan Growers Assn. 85:123-127.

Sanderlin, R.S. 1994. Effect of nut scab on pecan yield and quality components, p. 45-58. In: M.W Smith, W. Reid, and B.W. Wood (eds.). Sustaining pecan productivity into the $21^{\text {st }}$ century. Proc. USDA-ARS $2^{\text {nd }}$ Natl. Pecan Wkshp.

Sparks, D. 1992. Pecan cultivars: The Orchards Foundation. Pecan Prod. Innovations, Watkinsville, Ga.

Thompson, T.E. and L.J. Grauke. 1994. Genetic resistance to scab disease in pecan. HortScience 29:1078-1084.

Turechek, WW and K L Stevenson 1998. Effect of host resistance, temperature, leaf wetness, and leaf age on infection and lesion development of pecan scab. Phytopathology 88:1294-1301.

Vanderplank, J.E. 1984. Disease resistance in plants. Academic Press, Orlando, Fla.

Wolfe, M.S. 1993. Can the strategic use of disease resistant hosts protect inherent durability? p. 83-96. In: Th. Jacobs and J.E. Parleviet (eds.). Durability of disease resistance. Kluwer, Dordrecht. 